\title{
A Prospective Study of the Safety of Flexible Bronchoscopy under Propofol Sedation in Patients with and without Chronic Obstructive Pulmonary Disease
}

\author{
Marco Solis*, Marcos Hernandez, Cesar Duran, Roberto Dure and Silvia Quadrelli
}

Sanatorio Guemes University Hospital, Buenos Aires, Argentina

\begin{abstract}
Background and objective: The British Thoracic Society (BTS) recommends that sedation for fiber-optic bronchoscopy (FOB) should be offered to all patients. This study evaluates the safety of FOB under sedation in patients with COPD.

Methods: Is a prospective observational study, with the approval of institutional review board. Sedation was administered by a board-certified anaesthetist. Patients were premedicated (IV) with metoclopramide $10 \mathrm{mg}$, fentanyl initial dose: $25 \mu \mathrm{g}$, midazolam initial dose $2-3 \mathrm{mg}$. After an initial $50 \mathrm{mg}$ IV propofol, the dose was then carefully titrated according to the ASA physical status classification.

Results: The duration of the procedure was not different between the two groups ( $14.7 \pm 3.551 \mathrm{vs.} 14.9 \pm 3.8 \mathrm{~min}$ $p=0.695)$. Serious complications were very infrequent in both groups $(2.1 \mathrm{vs} .0 .07 \%, p=0.148)$. In the group of COPD patients there was no correlation between the lowest $\mathrm{SaO}_{2}$ during the procedure $(r=0.03, p=0.518)$ or the $\mathrm{SaO}_{2}$ at the end of the procedure $(r=-0.006, p=0.909)$ and the baseline $\mathrm{FEV}_{1}$. Neither the presence of a fall in the $\mathrm{SaO}_{2}$ greater than 4 points (HR 0.895, IC 0.452-1.773, $\mathrm{p}=0.750)$ nor a SaO lower than $90 \%$ during the procedure (HR 0.346 , IC $0.060-1.918, p=0.233$ ) or the general rate of complications (including desaturation) (HR: 0.627, $\mathrm{Cl}: 0.257-1.529$, $\mathrm{p}=0.305$ ) were predicted by a baseline $\mathrm{FEV}$, lower than $50 \%$.
\end{abstract}

Conclusions: We conclude that $\mathrm{FOB}$ under conscious sedation by a certified anesthesiologist is a safe procedure in patients with COPD with a low incidence of adverse effects.

Keywords: Bronchoscopy and interventional techniques; COPD; Sedation; Bronchoalveolar lavage; Desaturation; Ventilation

Abbreviations: COPD: Chronic Obstructive Pulmonary Disease; FOB: Fiberoptic Bronchoscopy; ASA: American Society of Anaesthesiologist

\section{Introduction}

COPD is a highly prevalent condition all over the world and its prevalence is increasing. Patients with COPD have comorbid illnesses or symptoms that often prompt bronchoscopic examination. These include chronic cough, hemoptysis, bronchiectasis, and a smoking history that increases risk for lung cancer. Bronchiectasis is a common cause of haemoptysis and seem to be more prevalent in COPD [1]. Herth et al. [2] had reported in a group of 135 patients having haemoptysis of unknown origin that $59 \%$ of patients were active smokers and $42 \%$ had COPD. Additionally, the development of minimally invasive bronchoscopic techniques as one-way bronchial valves inserted via fiberoptic bronchoscopy has widened the therapeutic use of bronchoscopy in COPD.

The British Thoracic Society recommends that sedation for flexible bronchoscopy should be offered to all patients in the absence of contraindication [3]. A survey of registered members of the British Thoracic Society, reported that $95 \%$ of centres routinely perform sedated bronchoscopy [4].

However, in spite of the higher prevalence of COPD in any bronchoscopy unit, the safety of this examination in this group of patients under sedation has received little attention. We designed this prospective observational study to evaluate the safety of FOB under the administration of propofol plus fentanyl for conscious sedation in patients with COPD. This study does not compare with others sedation approach.

\section{Material and Methods}

Prospective data of 598 patients from 2010 to 2016, undergoing elective diagnostic flexible bronchoscopy at a single tertiary referral hospital were analysed. 190 patients were diagnosed as COPD. The approval of the institutional review board was obtained. Emergency procedures, bronchoscopies performed bedside in the intensive care unit, therapeutic procedures, transbronchial needle aspiration, endobronchial ultrasound, and bronchoscopy studies performed only with local anesthesia or under general anesthesia were excluded. Patients with known allergy or intolerance to propofol or midazolam, pregnant or breastfeeding females were not included in the study. Informed consent was obtained from each patient and the study was approved by the institutional review board. In the procedures included, conscious sedation was achieved following the routine practice in the institution with intermittent boluses of intravenous midazolam and fentanyl.

All patients were assessed by a certified anesthesiologist and a chest physician prior to the procedure. Comorbidities and current medication such as anticoagulants, antiplatelet drugs, sedatives and hypnotics were

*Corresponding author: Marco Solis Malabia, Sanatorio Guemes University Hospital, Buenos Aires, Argentina, Tel: 541160003392; Fax: 541148211117; E-mail: msolisaramayo@yahoo.com.ar

Received November 20, 2017; Accepted November 23, 2017; Published November 30, 2017

Citation: Solis M, Hernandez M, Duran C, Dure R, Quadrelli S (2017) A Prospective Study of the Safety of Flexible Bronchoscopy under Propofol Sedation in Patients with and without Chronic Obstructive Pulmonary Disease. J Pulm Respir Med 7: 431. doi: 10.4172/2161-105X.1000431

Copyright: $\odot 2017$ Solis M, et al. This is an open-access article distributed under the terms of the Creative Commons Attribution License, which permits unrestricted use, distribution, and reproduction in any medium, provided the original author and source are credited. 
recorded. The anesthesiologist classified the patient as either low (ASAIII), medium (ASA III), or high risk (ASA IV-V) for the procedure according to the American Society of Anaesthesiologists (ASA) criteria. Patients were classified as COPD or non-COPD according to lung function performed within one week prior to bronchoscopy, and in the COPD group of patients, we classified between $\mathrm{FEV}_{1}<50 \%$ and $\mathrm{FEV}_{1}>50 \%$ to compare the results.

Bronchoscopy procedures were performed transnasally or transorally, with the patients in the semi-recumbent position, by an experienced bronchoscopist (official training of the Argentinean Association of Bronchology) or one of the 4 senior pulmonary fellows under close supervision of the attending physician.

Electrocardiographic and transcutaneous pulse oxymetric monitoring was recorded continuously during the procedure and automated non-invasive blood pressure was monitored every 5 minutes. All patients received supplemental oxygen at $41 / \mathrm{min}$ via nasal cannula which was increased if required to maintain oxygen saturation above $90 \%$.

Topical anaesthesia was achieved by application of a topical anaesthetic (10\% lidocaine spray) to the oropharynx. Nasal anaesthesia was achieved by $2 \%$ lidocaine gel, the instillation of $3 \mathrm{ml}$ aliquots of $1 \%$ lidocaine over the vocal cords and on the trachea and both right and left main bronchi. Instilled lidocaine doses were recorded for each patient. Supplemental doses of local anaesthesia were administered if required, as judged by the bronchoscopist and were recorded for each patient. No inhaled lidocaine was used for topical anaesthesia.

Sedation was administered by a board-certified anaesthetist. Patients were premedicated (IV) as a protocol of pre-sedation, with metoclopramide $10 \mathrm{mg}$, fentanyl initial dose: $25 \mu \mathrm{g}$, midazolam initial dose 2-3 mg. Loading doses of propofol were titrated to achieve adequate conscious sedation (onset of ptosis for bronchoscopy). Thereafter, conscious sedation was achieved with an IV infusion in an intermittent bolus technique, until reach a level 3 on de Ramsay sedative scale. After an initial $50 \mathrm{mg}$ IV propofol (sedation dose of 0.5-1 $\mathrm{mg} / \mathrm{kg}$-1 over 1-5 $\mathrm{min}$ ), the dose was then carefully titrated according to the ASA physical status classification: for ASA I and II, IV propofol boluses of 30-50 mg IV were applied, whereas for ASA III and IV, precisely $20 \mathrm{mg}$ propofol IV was administered based on the clinical response. Between each bolus, a pause lasting $\geq 30 \mathrm{~s}$ had to be observed. If the effect disappeared during the examination, additional IV boluses of 20-30 mg of propofol were given, depending on the clinical effect, to maintain the required level of sedation. Signs of pain or discomfort, agitation, persistent cough and inadequate motor or verbal response to manipulation were considered indicators for insufficient sedation, leading to administration of an additional dose of propofol (20-30 mg). The total dose of propofol was documented for each patient. All the patients received the same doses and protocol of sedation.

As a stated by the Canadian Association of Gastroenterology, the advantages or propofol over standard agents used for conscious sedation (eg. benzodiazepines and opiates) include [5]:

- A shorter time to recovery and, hence, earlier discharge from the endoscopy unit (3-5) patients who receive propofol (half-life 2 $\mathrm{min}$ to $4 \mathrm{~min}$ ) as a single agent recover normal neurological and social functioning significantly quicker than benzodiazepines (half-life $30 \mathrm{~min}$ ) and/or narcotics (half-life $3 \mathrm{~h}$ to $4 \mathrm{~h}$ )

- A quicker onset of action and les patient discomfort; both or which benefit the endoscopist and the patients.

\section{- Less nausea and vomiting.}

There is some suggestion that the pharmacological profile of propofol may be particularly advantageous in which patient cooperation is critical like COPD patients who have more cough or hypoxemia. Potentially, the largest impact of propofol use will be related to faster recovery times and earlier discharges from the endoscopy unit [5].

Diagnostic procedures, (brushing, washings, bronchoalveolar lavage (BAL), endobronchial and transbronchial biopsy), were performed dependent upon the criteria of the physician performing the bronchoscopy. The duration and type of diagnostic procedures were documented for each patient at the end of FOB. Haemodynamic parameters and complications were recorded on a form specifically designed for the study. Complications were defined as oxygen desaturation $\leq 90 \%$, hypotension with a systolic blood pressure of $<90 \mathrm{mmHg}$, fever, severe cough, pneumothorax and minor bleeding. Severe complications were defined as major bleeding, need to abort bronchoscopy, need for intubation, need for ICU transfer postbronchoscopy and death.

Differences in dichotomous variables were evaluated using the Chi-squared test or Fisher's exact test, as appropriate. Normally distributed parameters were analysed using an unpaired t-test for equality of means. Non-normally distributed parameters were analysed by nonparametric tests. All test were two-tailed; a p-value of $<0.05$ was considered significant. Univariate logistic regression analysis was used to identify COPD-related risk factors for the development of complications. The Statistical Package for Social Sciences (SSPS, Inc.) version 15 for Windows program (SPSS, Chicago, IL, USA) was used.

\section{Results}

We included 598 bronchoscopies performed in 598 patients. One hundred and ninety patients $(31.8 \%)$ were diagnosed as COPD. Demographics and main indications of the whole population are shown in Table 1.

Patients with COPD were older, showed a lower $\mathrm{FEV}_{1}$ and $\mathrm{FEV}_{1} /$ FVC ratio and had lower baseline oxygen saturation $\left(\mathrm{SaO}_{2}\right)$ (Table 2). An abnormal imaging study was a more frequent indication in COPD patients whilst suspicion of infection or immunosuppression was less common indications for the bronchoscopy amongst those patients (Table 2). The duration of the procedure was not different between the two groups $(14.7 \pm 3.551$ vs. $14.9 \pm 3.8 \mathrm{~min} \mathrm{p}=0.695)$. COPD patients were more frequently classified as ASA IV (11.2 vs. 9.4\%, $\mathrm{p}=0.011)$ (Table 3). Frequency of the performance of the different diagnostic procedures was not different in both groups (bronchoalveolar lavage 71.1 vs. $65 \%, \mathrm{p}=0.165$, transbronchial biopsy 45.4 vs. $46.0 \%, \mathrm{p}=0.490$ ).

\begin{tabular}{|c|c|}
\hline \multicolumn{2}{|l|}{ Demographics data of 598 patients } \\
\hline Age (years $\pm \mathrm{SD})$ & $54.4( \pm 13.76)$ \\
\hline COPD patients $\%(n)$ & $31.7 \%(n=190)$ \\
\hline $\mathrm{FEV}_{1} / \mathrm{FVC} \%(\% \pm \mathrm{SD})$ & $73.8 \%( \pm 15.1)$ \\
\hline $\mathrm{FEV}_{1} \%(\% \pm \mathrm{SD})$ & $75 \%( \pm 13.7)$ \\
\hline Hemoptysis \% (n) & $19.9 \%(n=119)$ \\
\hline Abnormal imaging \% (n) & $82.3 \%(n=492)$ \\
\hline Suspected infection \% (n) & $45.5 \%(n=272)$ \\
\hline Immunosuppressed \% (n) & $12.0 \%(n=72)$ \\
\hline Fall O2 saturation $>4$ points $\%(n)$ & $39.8 \%(n=238)$ \\
\hline Fall O2 saturation $>10$ points $\%(n)$ & $5.3 \%(n=32)$ \\
\hline Fall O2 saturation< $<90 \%(\mathrm{n})$ & $17.2 \%(n=103)$ \\
\hline Fall O2 saturation $<90 \%$ lasting more than one minute $\%(n)$ & $4.5 \%(n=27)$ \\
\hline Final saturation $<90 \% \%(\mathrm{n})$ & $0.33 \%(n=2)$ \\
\hline
\end{tabular}

Table 1: Demographics and main indications. 


\begin{tabular}{|c|c|c|c|c|c|}
\hline & \multicolumn{2}{|c|}{$\begin{array}{l}\text { COPD PATIENTS } \\
(n=109)\end{array}$} & \multicolumn{2}{|c|}{$\begin{array}{c}\text { NON COPD } \\
\text { PATIENTS }(n=408)\end{array}$} & \multirow[t]{2}{*}{$P$} \\
\hline & median & SD & Median & SD & \\
\hline Age (years) & 57.4 & \pm 11.73 & 53.0 & \pm 14.4 & $<0.001$ \\
\hline FEV $/$ /FVC (\%) & 53.8 & \pm 11.1 & 83.1 & \pm 7.8 & $<0.001$ \\
\hline $\mathrm{FEV}_{1}(\%)$ & 63.3 & \pm 15.9 & 81.2 & \pm 7.5 & $<0.001$ \\
\hline $\begin{array}{l}\text { Initial systolic BP } \\
(\mathrm{mmHg})\end{array}$ & 124.8 & \pm 11.5 & 124.0 & \pm 11.6 & 0.449 \\
\hline Final SBP $(\mathrm{mmHg})$ & 127.3 & \pm 15.6 & 128.4 & \pm 16.7 & 0.425 \\
\hline Fall SBP (mmHg) & 2.76 & \pm 16.4 & -4.42 & \pm 16.57 & 0.425 \\
\hline Initial SAT (\%) & 95.9 & \pm 2.3 & 96.6 & \pm 2.2 & 0.001 \\
\hline $\begin{array}{l}\text { Initial SAT with } \\
\mathrm{O}_{2}(\%)\end{array}$ & 91.8 & \pm 1.7 & 97.5 & \pm 1.2 & 0.083 \\
\hline Lowest SAT (\%) & 90.2 & \pm 4.4 & 93.0 & \pm 4.4 & $<0.001$ \\
\hline $\begin{array}{l}\text { Post-sedation SAT } \\
(\%)\end{array}$ & 94.2 & \pm 10.8 & 96.1 & \pm 2.6 & 0.001 \\
\hline Final SAT (\%) & 96.4 & \pm 3.5 & 97.5 & \pm 1.7 & $<0.001$ \\
\hline Fall SAT & 5.7 & \pm 4.73 & 3.6 & \pm 3.8 & $<0.001$ \\
\hline
\end{tabular}

Table 2: Baseline characteristic of patients.

\begin{tabular}{|l|c|c|c|}
\hline & COPD patients n (\%) & Non COPD patients n (\%) & P \\
\hline Hemoptysis & $30(15.8 \%)$ & $89(21.8 \%)$ & 0.052 \\
\hline Abnormal imaging & $143(75.3 \%)$ & $349(85.5 \%)$ & 0.002 \\
\hline Suspected infection & $68(35.8 \%)$ & $204(50 \%)$ & 0.001 \\
\hline Immunosuppressed & $15(7.9 \%)$ & $57(14 \%)$ & 0.021 \\
\hline
\end{tabular}

Table 3: Indication of bronchoscopy.

All the patients received propofol, the mean dose administrated was $227 \mathrm{mg}$; the mean doses of midazolam and fentanyl were $6.9 \mathrm{mg}$ and $110 \mathrm{mg}$, respectively. The mean time to sedation for propofol was $1.9 \mathrm{~min}$.

The fall of systolic blood pressure after sedation was not different in the 2 groups (Table 4) but oxygen saturation decreased significantly more in $\mathrm{COPD}$ patients and they reached a lower minimal $\mathrm{SaO}_{2}$ during the procedure (Table 4). Tolerance of the procedure was assessed by the patient more frequently as bad in the COPD group, but even amongst those patients that qualification was very infrequent $(4.8 v s$. $1.3 \% \mathrm{p}=0.012$ ). Complications other than $\mathrm{SaO}_{2}$ lower than $90 \%$ were not more frequent in COPD patients ( 7.4 vs. $11.8 \%, \mathrm{p}=0.064)$ but if the desaturation is included, the frequency of complications in COPD patients $v s$. no COPD patients, was significantly higher $(43.0$ vs. $6.9 \%$, $\mathrm{p}=>0.0001$ ). There was no difference in the prevalence of each single complication in COPD patients (Table 5). Serious complications were very infrequent in both groups ( 2.1 vs. $0.07 \%, \mathrm{p}=0.148)$. There were no deaths related to the procedure.

The decrease of $\mathrm{SaO}_{2}$ during the procedure was more frequent and profound in COPD patients (Table 6). However, a $\mathrm{SaO}_{2}$ below $90 \%$ after the finalisation of the procedure was a very infrequent event (1.1\%) and was not different between the two groups (Table 6).

In the group of COPD patients, comparing patients with $\mathrm{FEV}_{1}<50 \%$ vs. $\mathrm{FEV}_{1}>50 \%$, there was no correlation between the lowest $\mathrm{SaO}_{2}$ during the procedure $(\mathrm{r}=0.03, \mathrm{p}=0.518)$ or the $\mathrm{SaO}_{2}$ at the end of the procedure $(\mathrm{r}=-0.006, \mathrm{p}=0.909)$. Neither the presence of a fall in the $\mathrm{SaO}_{2}$ greater than 4 points (HR 0.895, IC 0.452-1.773, $\mathrm{p}=0.750$ ) nor a $\mathrm{SaO}_{2}$ lower than $90 \%$ during the procedure (HR: 0.346, IC: $0.060-1.918$, $\mathrm{p}=0.233$ ) or the general rate of complications (including desaturation) (HR: 0.627, CI: $0.257-1.529, \mathrm{p}=0.305$ ) were predicted by a baseline $\mathrm{FEV}_{1}$ lower than $50 \%$.

Full recovery occurred in $100 \%$ of non COPD patients versus $99.5 \%$ of COPD patients $60 \mathrm{~min}$ after the procedure. After the $2 \mathrm{~h}$ of routine observation in the post-procedures room, all return home without

\begin{tabular}{|l|c|c|}
\hline & COPD patients & Non COPD patients \\
\hline ASA 0 $\%$ & $0(0 \%)$ & $20(5.2 \%)$ \\
\hline ASA 1 $\%$ & $21(12.4 \%)$ & $47(12.3 \%)$ \\
\hline ASA 2 $\%$ & $46(27.2 \%)$ & $74(19.3 \%)$ \\
\hline ASA 3 $\%$ & $83(49.1 \%)$ & $206(53.8 \%)$ \\
\hline ASA 4 $\%$ & $19(11.2 \%)$ & $36(9.4 \%)$ \\
\hline
\end{tabular}

Table 4: ASA grade.

\begin{tabular}{|l|c|c|c|}
\hline & $\begin{array}{c}\text { COPD patients } \\
\mathbf{n}(\%)\end{array}$ & $\begin{array}{c}\text { Non COPD patients } \\
\mathbf{n}(\%)\end{array}$ & $\mathbf{P}$ \\
\hline Fever & $33(17.4 \%)$ & $89(21.8 \%)$ & 0.125 \\
\hline Pneumothorax & $2(1.1 \%)$ & $0(0 \%)$ & 0.101 \\
\hline Bleeding & $14(7.4 \%)$ & $35(8.6 \%)$ & 0.372 \\
\hline Cough & $1(0.5 \%)$ & $10(2.5 \%)$ & 0.089 \\
\hline SBP <90 mmHg & $34(17,9 \%)$ & $156(38.2 \%)$ & 0.463 \\
\hline \multicolumn{3}{|c|}{ Major complications } \\
\hline Major bleeding & $0(0 \%)$ & $1(1.17 \%)$ & 0.143 \\
\hline $\begin{array}{l}\text { Need to abort } \\
\text { bronchoscopy }\end{array}$ & $1(0.53 \%)$ & $1(1.17 \%)$ & 0.579 \\
\hline Need for intubation & $1(0.53 \%)$ & $0(0 \%)$ & 0.143 \\
\hline $\begin{array}{l}\text { Need for ICU } \\
\text { transfer post- } \\
\text { bronchoscopy }\end{array}$ & $2(1.05 \%)$ & $1(1.17 \%)$ & 0.193 \\
\hline
\end{tabular}

Table 5: Procedural complications.

\begin{tabular}{|l|c|c|c|}
\hline & COPD patients & $\begin{array}{c}\text { Non COPD } \\
\text { patients }\end{array}$ & P \\
\hline Fall $\mathrm{SaO}_{2}>4$ points $\mathrm{n}(\%)$ & $102(53.7 \%)$ & $136(33.3 \%)$ & $<0.001$ \\
\hline Fall $\mathrm{SaO}_{2}>10$ points $\mathrm{n}(\%)$ & $25(13.2 \%)$ & $7(1.7 \%)$ & $<0.001$ \\
\hline $\begin{array}{l}\text { Saturation } \\
\text { more than one minute } \mathrm{n}(\%)\end{array}$ & $23(12.10 \%)$ & $4(0.98 \%)$ & $<0.001$ \\
\hline Final saturation $<90 \% \mathrm{n}(\%)$ & $2(1.1 \%)$ & $0(0 \%)$ & 0.101 \\
\hline
\end{tabular}

Table 6: Oxygen saturation changes.

incidents, except for only one patient in the COPD group. A total of $99 \%$ of patients were willing to repeat the same procedure again.

\section{Discussion}

This study demonstrates that flexible bronchoscopy under conscious sedation can be safely performed in patients with COPD, even in those with severe airflow obstruction. Very early in the development of the flexible bronchoscopy era, it had been shown that fiberoptic bronchoscopy may cause a statistically significant but small impairment in lung mechanics and gas exchange in patients with chronic airways obstruction but that the small magnitude of those changes suggested the procedure was safe enough if adequate oxygenation is provided during the bronchoscopy [6,7]. Stoltz et al. [8] showed in 40 COPD patients that compared to prebronchoscopy values, postbronchoscopy $\mathrm{FEV}_{1}$ percentage of predicted decreased significantly in all patients, with or without premedication with salbutamol. Hattotuwa et al. [9] had previously shown in 57 COPD patients (mean baseline as \% of predicted: FEV 47.9\%) in who bronchoscopy was performed with research purposes, the frequent presence of falls in oxygen saturations (lowest $88 \%$ ) during the performance of BAL and with prolonged coughing. The authors remarked that those episodes of desaturation were transient and treated successfully with supplemental oxygen. They reported a $2.0 \%$ incidence of adverse events requiring hospital treatment and a $3.1 \%$ incidence of hemoptysis requiring no intervention with all the complications occurring in the moderate to severe COPD. Sedation is increasingly used to perform bronchoscopy. Although in 1983, a postal survey [10] of bronchoscopic practice in the United Kingdom indicated that of the 227 physicians performing fiberoptic 
bronchoscopy, only $6 \%$ did so without routinely using any sedative drugs, according to a later survey taken in 2003 amongst members of the British Thoracic Society, 95\% of centres routinely perform sedated bronchoscopy [4]. It has been reported that most patients (62\%) undergoing fiberoptic bronchoscopy are anxious and fear pain and breathing difficulties during the procedure [11]. The aim of sedation is to improve patient comfort and to alleviate patient anxiety, cough and dyspnoea which may in turn contribute to the reduction of the procedure-related complications [12]. It does not prevent the need of analgesia. Midazolam [13] is a water-soluble, short-acting benzodiazepine that does not produce pain or thrombophlebitis on injection and provides profound procedural amnesia. When used alone midazolam has a limited effect on cardiorespiratory function but combined with opioids, the respiratory depression effects of both classes of agents may be potentiated [14]. Although apnoea is not usually seen with low doses used for moderate sedation, it may occur with higher doses.

As benzodiazepines and propofol provide only sedation and amnesia but have a very weak analgesic effect, they are often used in conjunction with opioids. A common consequence of all these agents is respiratory depression, making crucial a careful management of the dose and the rate of administration [15].

Propofol (2,6-di-isopropylphenol), is a sedative hypnotic that has proved to be a safe option to combined sedation with midazolam due to its rapid onset of action and fast recovery time which adds the benefit of making possible a timely discharge [16]. However, unlike the benzodiazepines, propofol does not have a reversal agent. Randomised controlled studies of the use of propofol in bronchoscopy have shown that sedation with propofol compared to no sedation provides less cough, pain, sensation of asphyxiation and total amnesia with no differences in oxygen saturations between the groups [17].

Sipe et al. [18] showed that the time to sedation was faster in the propofol patients than in midazolam and meperidine $(2.1 \mathrm{~min} v s$. $7.0 \mathrm{~min} ; \mathrm{p}<0.0001)$ and depth of sedation was grather $(\mathrm{p}<0.0001)$. On average, after the procedure, the propofol patients could stand at the bedside sooner, reached full recovery faster and were discharged sooner (all $\mathrm{p}<0.0001$ ). Patients who received propofol also expressed greater overall mean satisfaction on a 10 point-point visual analog scale (9.3 vs. 8.6; $\mathrm{p}<0.05)$ neither group had serious complications.

There is no specific antidote for propofol. Caution is required during administration to avoid deep anaesthesia. Generally, a propofol loading dose of $40 \mathrm{mg}$ to $50 \mathrm{mg}$ is given with further smaller bolus loads to maintain sedation, with a typical total dose between $100 \mathrm{mg}$ and $300 \mathrm{mg}$.

The present study is important because demonstrates that receiving propofol using an intermittent bolus technique combined with fentanyl and midazolam in flexible bronchoscopy produces a safety sedation, faster to recovery the neurological status of the patients and the number of adverse events and complications is similar in patients with and without COPD. The feasibility and safety of propofol sedation as administered by repeated bolus technique has been reported in two large cohort studies of propofol sedation in flexible bronchoscopy $[16,17]$ but to our knowledge, this is the first trial comparing the administration of conscious sedation in patients with and without COPD.

In the current study, the frequency and severity of adverse events observed during flexible bronchoscopy was similar in patients with COPD, except for the presence of the fall in the oxygen saturation. Even when patients with COPD $(\mathrm{n}=190)$ were significantly older $(\mathrm{p}=<0.001)$, had poorer lung function $(\mathrm{p}=<0.001)$ and higher ASA-scores (ASA IV: 11.2 vs. 9.4\%), the serious adverse events were extremely infrequent in those patients (major bleeding, $n=0$, need to abort bronchoscopy: $n=1$, need for intubation: $\mathrm{n}=1$, need for ICU transfer post-bronchoscopy, $\mathrm{n}=2$ ) and with no difference with the non-COPD population.

The most frequent complication, in the 598 patients, was the fall of oxygen saturation $\left(\mathrm{SaO}_{2}\right)$. A fall of 4 points or more in the $\mathrm{SaO}_{2}$ occurred in almost $40 \%$ of patients and in $5.3 \%$ it was higher than 10 points. However only $4.5 \%$ of patients were under $90 \%$ for more than 60 seconds and less than $1 \%$ ended the procedure with a $\mathrm{SaO}_{2}$ lower than $90 \%$. Those figures are similar to those previously reported in patients receiving propofol in bolus or infusion [13,19-21] and slightly higher that the observed after the infusion of midazolam as single drug [22]. The frequency of fall in the $\mathrm{SaO}_{2}$ was the only adverse event more frequent in COPD patients. The median $\mathrm{SaO}_{2}$ after the administration of sedation (and before the beginning of the procedure) $(\mathrm{p}=0.001)$, the lowest $\mathrm{SaO}_{2}(\mathrm{p}=<0.001)$, the final $\mathrm{SaO}_{2}(\mathrm{p}=<0.001)$ and the magnitude of the oxygen saturation fall $(\mathrm{p}=<0.001)$ showed a deeper effect of sedation and the performance on the procedure on oxygen saturation in COPD patients. That similar prevalence of complications (other than transient fall in $\mathrm{SaO}_{2}$ ) in COPD, has been recently shown by Grendelmeier et al. [23] in a congress abstract that demonstrated that the incidence of complications (Chin support, artificial airway, intubation, bleeding, pneumothorax, need for abortion or ICU, death) were similar in both groups. In their series they also found a lower lowest $\mathrm{SO}_{2}(85 \%$ vs. $87 \%, \mathrm{p}=0.004)$ during examination in COPD patients. The level of desaturation was much more pronounced than in our patients, but they included complex interventions such as EBUS, stent-placement, laser and bronchoscopy lung volume reduction procedures that were excluded in our study. Probably because of that, their COPD patients had a longer examination time (differently from our series) and as results were not adjusted to the presence of a therapeutic procedure or the time of examination, differences may be based on a higher frequency of more complicated procedures in COPD patients.

Interestingly, the initial $\mathrm{FEV}_{1}$ was not able to predict the rate of complications or even the magnitude of the fall in $\mathrm{SaO}_{2}$. It is not different from the limitations of $\mathrm{FEV}$ as a predictor of risk in other non-resection surgical procedures [24].

\section{Conclusion}

We have shown in this prospective study a $7.4 \%$ incidence of adverse events but only $2 \%$ of serious adverse events (only $1 \%$ requiring hospitalization) in a group of patients with COPD including subjects with severe disease. We conclude that fiberoptic bronchoscopy under conscious sedation by a certified anesthesiologist is a safe procedures in patients with COPD with a low incidence of adverse effects in spite of a very low $\mathrm{FEV}_{1}$.

\section{References}

1. Patel IS, Vlahos I, Wilkinson TMA, Lloyd-Owen SJ, Donaldson GC, et al. (2004) Bronchiectasis, exacerbation indices, and inflammation in chronic obstructive pulmonary disease. Am J Respir Crit Care Med 170: 400-407.

2. Herth F, Ernst A, Becker HD (2001) Long-term outcome and lung cancer incidence in patients with hemoptysis of unknown origin. Chest 120: 1592 1594.

3. Honeybourne D, Babb J, Bowie P, Brewin A, Fraise A, et al. British Thoracic Society guidelines on diagnostic flexible bronchoscopy. Thorax 56: I11-I21.

4. Pickles J, Jeffrey M, Datta A, Jeffrey AA (2003) Is preparation for bronchoscopy optimal? Eur Respir J 22: 203-206.

5. Byrne BF, Chiba N, Singh H, Sadowski DC (2008) Propofol use for sedation 
Citation: Solis M, Hernandez M, Duran C, Dure R, Quadrelli S (2017) A Prospective Study of the Safety of Flexible Bronchoscopy under Propofol Sedation in Patients with and without Chronic Obstructive Pulmonary Disease. J Pulm Respir Med 7: 431. doi: 10.4172/2161-105X.1000431

Page 5 of 5

during endoscopy in adults: A Canadian Association of Gastroenterology position statement. Can J Gastroenterol 22: 457-459.

6. Salisbury BG, Metzger LF, Altose MD, Stanley NN, Cherniack NS (1975) Effect of fiberoptic bronchoscopy on respiratory performance in patients with chronic airways obstruction. Thorax 30: 441-446.

7. Busse WW, Wanner A, Adams K, Reynolds HY, Castro M, et al. (2005) Investigative bronchoprovocation and bronchoscopy in airway diseases. Am J Respir Crit Care Med 172: 807-816

8. Stolz D, Pollak V, Chhajed PN, Gysin C, Pflimlin E, et al. (2007) A randomized, placebo-controlled trial of bronchodilators for bronchoscopy in patients with COPD. Chest 131: 765-772.

9. Hattotuwa K, Gamble EA, O'Shaughnessy T, Jeffery PK, Barnes NC (2002) Safety of bronchoscopy, biopsy, and BAL in research patients with COPD. Chest 122: 1909-1912.

10. Simpson FG, Arnold AG, Purvis A, Belfield PW, Muers MF, et al. (1986) Postal survey of bronchoscopic practice by physicians in the United Kingdom. Thorax 41: 311-317.

11. Poi PJH, Chuah SY, Srinivas P, Liam CK (1998) Common fears of patients undergoing bronchoscopy. Eur Repir J 11: 1147-1149.

12. Matot I, Kramer MR (2000) Sedation in outpatient bronchoscopy. Resp Med 94: 1145-1153.

13. Clarkson K, Power CK, O'Connell F, Pathmakanthan S, Burke CM (1993) A comparative evaluation of propofol and midazolam as sedative agents in fiberoptic bronchoscopy. Chest 104: 1029-1031.

14. Greig JH, Cooper SM, Kasimbazi HJN, Monie RDH, Fennerty AG, et al. (1995) Sedaton for fibre-optic bronchoscopy. Respir Med 89: 53-56.

15. Bailey PL, Stanley TH (1990) Narcotic intravenous anesthetics.
16. Clark G, Licker M, Younossian AB, Soccal PM, Frey JG, et al. (2009) Titrated sedation with propofol or midazolam for flexible bronchoscopy: A randomised trial. Eur Respir J 34: 1277-1283.

17. Gonzalez R, De-La-Rosa-Ramirez I, Maldonado-Hernandez A, DominguezCherit G (2003) Should patients undergoing a bronchoscopy be sedated? Acta Anaesthesiol Scand 47: 411-415.

18. Sipe BW, Rex DK, Latinovich D, Overley C, Kinser K, et al. (2002) Propofo versus midazolam/meperidine for outpatient colonoscopy: Administration by nurses supervised by endoscopists. Gastrointest Endos 55: 815-825.

19. Bosslet GT, DeVito ML, Lahm T, Sheski FD, Mathur PN (2010) Nurseadministered propofol sedation: Feasibility and safety in bronchoscopy. Respiration 79: 315-321.

20. Grendelmeier P, Kurer G, Pflimlin E, Tamm M, Stolz D (2011) Feasibility and safety of propofol sedation in flexible bronchoscopy. Swiss Med Wkly 141 w13248.

21. Schlatter L, Pflimlin E, Fehrke B, Meyer A, Tamm M, et al. (2011) Propofol versus propofol plus hydrocodone for flexible bronchoscopy: A randomised study. Eur Respir J 38: 529-537.

22. Contoli M, Gnesini G, Artioli D, Caterina R, Sferra S, et al. (2013) Midazolam in flexible bronchoscopy premedication: Effects on patient-related and procedurerelated outcomes. J Bronchology Interv Pulmonol 20: 232-240.

23. Grendelmeier P, Tamm M, Jahn K, Pflimlin E, Stolz D (2015) Flexible bronchoscopy (FB) in COPD: A prospective, case-control study. Pneumologie 69: P25.

24. Wong DH, Weber EC, Schell MJ, Wong AB, Anderson CT, et al. (1995) Factors associated with postoperative pulmonary complications in patients with severe chronic obstructive pulmonary disease. Anesth Analg 80: 276-284. 